\title{
Ischemia Alters the Expression of Connexins in the Aged Human Brain
}

\author{
Taizen Nakase, ${ }^{1}$ Tetsuya Maeda, ${ }^{2}$ Yasuji Yoshida, ${ }^{3}$ and Ken Nagata ${ }^{2}$ \\ ${ }^{1}$ Department of Stroke Science, Research Institute for Brain \& Blood Vessels-Akita, 6-10 Senshu Kubota Machi, Akita 010-0874, Japan \\ ${ }^{2}$ Department of Neurology, Research Institute for Brain \& Blood Vessels-Akita, 6-10 Senshu Kubota Machi, Akita 010-0874, Japan \\ ${ }^{3}$ Department of Pathology, Research Institute for Brain \& Blood Vessels-Akita, 6-10 Senshu Kubota Machi, Akita 010-0874, Japan
}

Correspondence should be addressed to Taizen Nakase, nakase@akita-noken.go.jp

Received 6 April 2009; Revised 8 June 2009; Accepted 30 June 2009

Recommended by Thomas van Groen

\begin{abstract}
Although the function of astrocytic gap junctions under ischemia is still under debate, increased expression of connexin 43 (Cx43) has been observed in ischemic brain lesions, suggesting that astrocytic gap junctions could provide neuronal protection against ischemic insult. Moreover, different connexin subtypes may play different roles in pathological conditions. We used immunohistochemical analysis to investigate alterations in the expression of connexin subtypes in human stroke brains. Seven samples, sectioned after brain embolic stroke, were used for the analysis. Data, evaluated semiquantitatively by computer-assisted densitometry, was compared between the intact hemisphere and ischemic lesions. The results showed that the coexpression of Cx32 and $\mathrm{Cx} 45$ with neuronal markers was significantly increased in ischemic lesions. $\mathrm{Cx} 43$ expression was significantly increased in the colocalization with astrocytes and relatively increased in the colocalization with neuronal marker in ischemic lesions. Therefore, $\mathrm{Cx} 32, \mathrm{Cx} 43$, and $\mathrm{Cx} 45$ may respond differently to ischemic insult in terms of neuroprotection.
\end{abstract}

Copyright (C) 2009 Taizen Nakase et al. This is an open access article distributed under the Creative Commons Attribution License, which permits unrestricted use, distribution, and reproduction in any medium, provided the original work is properly cited.

\section{Introduction}

Astrocytes are a major cell type in the central nervous system and play an important role in regulating brain metabolism $[1,2]$. Moreover, astrocytes compose the frame network and communicate through gap junctions. A gap junction hemichannel consists of six connexins, and the connexin $43(\mathrm{Cx} 43)$ is a main subtype of astrocytic gap junctions $[3,4]$. Previous studies showed that heterozygous Cx43 null mice exhibited significantly increased infarct volume [5] and enhanced apoptosis following ischemic brain insult [6]. Likewise, mice lacking Cx43 in astrocytes showed a significantly increased infarct volume and amplified inflammatory response in the penumbra compared to control littermates [7]. These results suggested that astrocytic gap junctions may play a critical role in controlling neuronal apoptosis and inflammatory response following brain ischemia. Moreover, in ischemic brain lesions of human samples, the expressed level of Cx43 was significantly increased in the penumbra compared to the intact area [8]. Therefore, astrocytic gap junctions may provide neuronal protection against ischemic insult not only in animal models but also in the human brain. However, the effects of astrocytic gap junctions on pathological conditions are still being debated [9, 10]. Recently, gap junctions composed of different types of connexins have been reported to have selective permeability for different biological molecules [11]. A few reports have also reported alterations of connexin expression under pathological conditions in the human brain [12, 13].

In this study, we used immunohistochemical analysis to examine the levels of $\mathrm{Cx} 26, \mathrm{Cx} 32, \mathrm{Cx} 43$, and $\mathrm{Cx} 45$ expression in neurons and astrocytes in ischemic lesions from the view point of human brain pathology.

\section{Materials and Methods}

2.1. Samples. We screened postmortem human brain samples with ischemic stroke $(n=53)$ in our hospital. First, we carefully checked all clinical records and image examinations, such as CT, MRI, MRA, and ultrasound echo cardiography 
TABLE 1: Background of all cases. F: female, M: male, lt.: left, rt.: right, MCA: middle cerebral artery, PCA: posterior cerebral artery, ACA: anterior cerebral artery, ICA: internal carotid artery.

\begin{tabular}{lccccc}
\hline No. & Age & Sex & Symptoms & Lesions & Duration to death \\
\hline$(1)$ & 76 & F & sudden onset, lt. hemiparesis & Rt. MCA \& PCA territories & 4 days, 3 hours \\
$(2)$ & 74 & M & sudden onset, lt. hemiparesis & Rt. ACA \& MCA territories & 2 days, 5 hours \\
$(3)$ & 70 & F & sudden onset, lt. hemiparesis & Rt. ICA territory & 4 days, 3 hours \\
$(4)$ & 73 & M & sudden onset, lt. hemiparesis & Rt. MCA territory & 5 days, 18 hours \\
$(5)$ & 87 & F & sudden onset, rt. hemiparesis & Lt. MCA territory & 6 days, 5 hours \\
$(6)$ & 82 & M & sudden onset, rt. hemiparesis & Lt. ACA \& MCA territories & 5 days, 12 hours \\
$(7)$ & 77 & F & sudden onset, rt. hemiparesis & Lt. MCA territory & 6 days \\
\hline Ave. & $77 \pm 5.8$ & & & & \\
\hline
\end{tabular}

of all sample cases. Embolic infarction brains sectioned after stroke were recruited in this study. Because of restricting artifacts in the pathological analysis, we selected only those cases with atrial fibrillation (Af) as the embolic source. They were sectioned within one week after hospitalization. Then, 7 cases were well preserved and fit our criteria for pathological observation. Precise clinical data are shown in Table 1.

2.2. Immunohistochemistry. The samples were fixed in phosphate buffered paraformaldehyde for one month and embedded in paraffin blocks. We observed whole brain sections with hematoxyline and eosin (H\&E) staining under photomicroscope (Eclipse E800, Nikon, Tokyo, Japan). Then, smaller brain samples (approximately $3 \mathrm{~cm} \times 2 \mathrm{~cm}$ ) were sectioned from the intact and ischemic cortex and included surrounding areas as shown in the representative picture (Figure 1). These brain sections ( $5 \mu \mathrm{m}$ in thickness) were mounted on glass slides and deparaffinized with xylene and graded ethanol solutions. Sections were then immersed in methanol with $0.3 \% \mathrm{H}_{2} \mathrm{O}_{2}$ for 15 minutes to inactivate internal peroxidase. After the slides were treated with $0.1 \%$ trypsin for 5 minutes, they were irradiated with microwaves in $0.01 \mathrm{M}$ citrate buffer $(\mathrm{pH} 6.0)$ for 5 minutes at $500 \mathrm{~W}$, followed by 20 minutes cooling for antigen-retrieval. The samples were then blocked with Protein Block Serum-Free (X0909, Dako, Glostrup, Denmark) for 30 minutes at room temperature. The sections were incubated with glial fibrillary acidic protein (GFAP: monoclonal, Dako), microtubuleassociated protein 2 (MAP2: monoclonal, Chemicon, Temecula, CA), connexin 26 (Cx26: polyclonal, Chemicon), Cx32 (polyclonal, ProteinTech Group Inc., Chicago, IL), Cx43 (polyclonal, Chemicon), and Cx45 (polyclonal, Alpha diagnostic Intl., San Antonio, TX) antibodies (dilution was 1 : 500, $1: 1000,1: 500,1: 200,1: 500$, and 1 : 400 , resp.) in $0.3 \%$ Tween 20 in PBS overnight at $4^{\circ} \mathrm{C}$, then reacted with appropriate secondary antibodies (Alexa Fluor, Molecular Probes Inc., Eugene, OR) in 0.3\% Tween20 in PBS for 30 minutes at room temperature. As for the primary antibodies, GFAP was used for the detection of astrocytes, and MAP2 was used as the neuronal marker. The sections were observed under a luminescence microscope (Nikon Eclipse E600) and images captured with an attached digital camera (DXM1200C, Nikon).

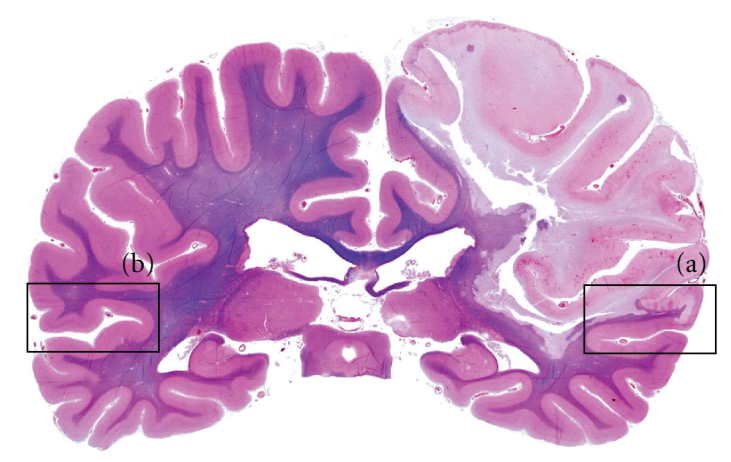

FIGURE 1: Representative picture of brain slice stained with H\&E. Pale colored area shows the infarct lesion. Rectangle lined areas indicate the selected regions of interest (ischemic cortex (a) and intact cortex (b)).

2.3. Morphometry. Because we were not able to evaluate the exact amount of protein in tissue which was used in this study, we analyzed the amount of protein expression semiquantitatively. That is, the area of immunoreactive plaques was measured in four randomly selected regions $(220 \mu \mathrm{m}$ $\times 165 \mu \mathrm{m}$ square) and comparisons made between intact and ischemic peripheral areas. The analysis was performed with the aid of computer software (Scion Image Beta 4.0.2, Scion Image Co., Frederick, MD). Pixel counts were used for calculation. All measurements were repeated on two different slides for each patient. On the pictures of double immunohistochemical staining, yellow plaques represent the coexpression of connexin proteins and neuronal or astrocytic markers. These yellow plaques were extracted with the assistance of computer software (Adobe Photoshop CS2, Adobe Systems Inc., San Jose, CA), to assess the amount of colocalized connexins. All acquired images were standardized by subtracting background density; that is, particles with sizes smaller than 50 pixels or larger than 500 pixels were excluded from images in our samples, as they are staining debris confirmed by negative controls.

2.4. Statistical Analysis. All data is presented as mean $\pm \mathrm{SD}$ values for each model. Mean values in each lesion were compared by an ANOVA posthoc test, Bonferroni/Dunn method using Stat View J 5.0 software (SAS Institute Inc., Cary, NC). Values of $P<.05$ were considered as significant. 


\section{Results and Discussion}

We observed the expression of different types of connexins in neurons and astrocytes to assess whether connexins play different roles under ischemic conditions. In this study, the expression of $\mathrm{Cx} 26, \mathrm{Cx} 32, \mathrm{Cx} 43$, and $\mathrm{Cx} 45$ was investigated because of previous reports in which critical roles for these connexins were suggested. $\mathrm{Cx} 26$ has been reported to form a major component of astrocytic gap junctions [14, 15], although its presence and function in the brain is still under debate $[16,17]$. Hemichannels formed by $\mathrm{Cx} 26$ and $\mathrm{Cx} 32$ have been reported to pass cGMP more effectively than Cx32 homomeric hemichannels, suggesting that the effect of $\mathrm{Cx} 26$ is specific in cGMP transport [18]. Cx32 is expressed not only in gap junctions of Schwann cells [19] but also in neurons and oligodendrocytes in the central nervous system (CNS) $[20,21]$. Cx32 was reported to participate in regulating the permeability of adenosine, a critical component of ATP [22]. Moreover, Cx32 knockout mice presented amplified neuronal loss following brain ischemia as compared to wild type mice [23]. Cx45 is reported to be expressed in neuronal and oligodendrocytic gap junctions [24, 25]. Electrical synapses composed of gap junctions, in which the main subtype of connexin is Cx45 [26], could disrupt chemical neurotransmission during development and regeneration [27]. However, it has also been reported that a $\mathrm{Ca}^{2+}$ influx has a critical impact on spreading cell damage from the core lesion to the penumbral area after ischemia $[28,29]$. Therefore, the role of electrical synapses composed of gap junctions against ischemic insult is still controversial. Cx43 is a major component of astrocytic gap junctions [3, 4]. We have reported that the reduction of $\mathrm{Cx} 43$ expression extended neuronal apoptosis and amplified gliosis following ischemia [7, 30]. Moreover, gap junctions composed of Cx43 are reported to be involved in the distribution of ATP between attached cells [22].

The immunohistochemical staining revealed that the gross expression of Cx32 (Figures 2(a) and 2(b)) was relatively increased in the ischemic area compared to the intact area (Figure 2(g): $P=.051)$. As seen in Figures 2(c) and 2(d), the expression of the yellow plaques representing Cx32 showed colocalization with MAP2. Expression was visualized along neuronal axons and was more abundant in the ischemic lesion than those in the intact area. According to calculations of the amount of the yellow plaques, Cx32 expression in MAP2 positive areas was significantly increased in the ischemic lesion compared to the intact area (Figure $2(\mathrm{~g}), P=.009)$. On the contrary, the expression of Cx32 on GFAP positive cells was not changed in the ischemic lesion (Figures 2(e), 2(f), and 2(g)).

The expression of $\mathrm{Cx} 45$ (Figures 3(a) and 3(b)) was significantly increased in ischemic lesions compared to intact area (Figure $3(\mathrm{~g}), P=.0003)$. Cx45 expression colocalized in MAP2 positive area was observed in neuronal body and axons (Figures 3(c) and 3(d)) and was significantly increased in the ischemic lesion compared to the intact area (Figure 3(g), $P=.0047)$. As shown in Figures 3(e) and 3(f), the expression of Cx45 in GFAP positive cells was not changed between ischemic and intact regions (Figure 3(g)).
As shown in Figures 4(a) and 4(b), the gross expression of Cx43 was significantly increased in ischemic lesions compared to intact tissue (Figure $4(\mathrm{~g}), P=.0154)$ ). The coexpression of Cx43 and MAP2 (Figures 4(c) and 4(d)) was visualized as punctate staining and relatively increased in the ischemic lesion compared to intact area (Figure $4(\mathrm{~g})$, $P=.0599$ ). The coexpression of $\mathrm{Cx} 43$ and GFAP (Figures $4(\mathrm{e})$ and $4(\mathrm{f}))$ was significantly increased in ischemic lesions compared to intact area (Figure $4(\mathrm{~g}), P<.001$ ). The results supported previous reports in which showed increased $\mathrm{Cx} 43$ expression in the ischemic lesion $[8,31$, 32].

Meanwhile, the gross expression of Cx26 was not changed between the intact area and ischemic lesion. The amount of Cx26 expression which colocalized with MAP2 or GFAP positive area revealed no significant difference between intact and ischemic regions (Figure 5). Since the role of $\mathrm{Cx} 26$ in astrocytic gap junctions is still controversial, our findings may support previous reports [17].

In the ischemic lesion, neurons and astrocytes are perishing, and their cell bodies and processes will shrink. On the other hand, some astrocytes and microglia react by expanding their body and processes as an inflammatory response. As mentioned above, both $\mathrm{Cx} 32$ and $\mathrm{Cx} 45$ exist in neurons and/or oligodendrocytes, and $\mathrm{Cx} 43$ exists in astrocytes. In this study, a significant increase of $\mathrm{Cx} 32$ and Cx45 expression was mostly observed in neuronal axons in ischemic lesions. In the CNS, the neuronal axon is covered with a myelin sheath of oligodendrocytes, and therefore, the expression of $\mathrm{Cx} 32$ and $\mathrm{Cx} 45$ could be increased in either, or both, neuronal axons and oligodendrocytes after ischemic insult. The Cx43 expression was significantly increased in astrocytes within ischemic lesions and was slightly increased in the coexpression of Cx43 and MAP2 after ischemic insult, suggesting that $\mathrm{Cx} 43$ may participate in the composition of gap junctions between neurons and astrocytes activated by ischemic stress. Since $\mathrm{Cx} 32$ relates to adenosine transport and $\mathrm{Cx} 43$ participates in distributing ATP, both $\mathrm{Cx} 32$ and Cx43 may play an important role in maintaining energy support after ischemic insult. $\mathrm{Cx} 45$ has been reported to play a role in spreading chemical stress against neurons from the ischemic core to peripheral area. It would require further assessment to reveal the effect of increased Cx45 expression following ischemia.

We could not assess the expression of Cx36, one of the major components of neuronal gap junctions, because our samples, fixed in paraformaldehyde and embedded in paraffin blocks, were unable to be stained with any antiCx36 antibodies we had available. Cx30 is also a major connexin subtype in astrocytic gap junctions. However, the anti-Cx30 antibodies which were used in this study did not show reactivity against human brain samples. Since the evidence showing that $\mathrm{Cx} 30$ may compensate for the lack of $\mathrm{Cx} 43$ has been accumulating $[6,17]$, the alteration of Cx30 expression after ischemic stress should be explored. Moreover, we had not directly observed gap junctions between neurons and astrocytes. Alternatively, double immunohistochemical staining was used for investigating the expression of specific connexins in neurons and 


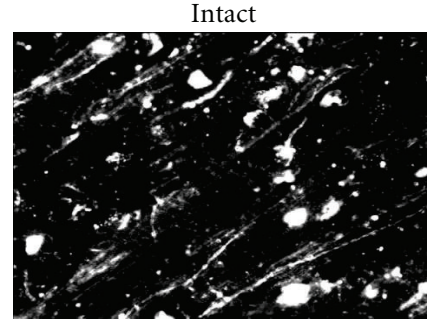

(a)

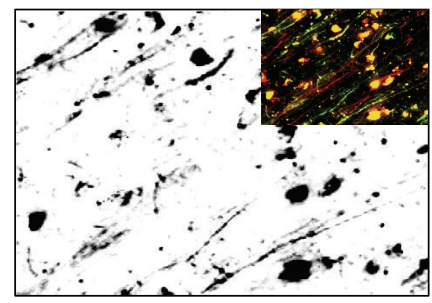

(c)

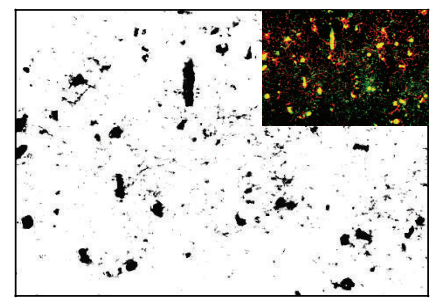

(e)

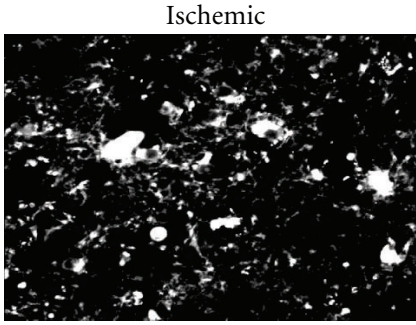

(b)

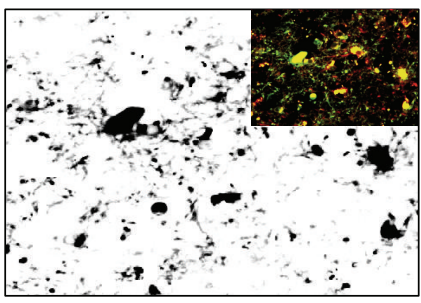

(d)

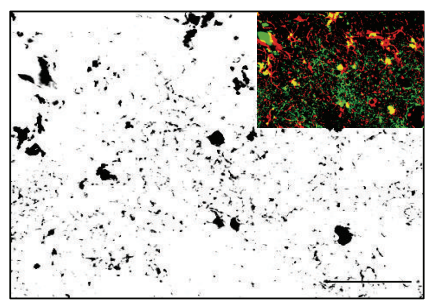

(f)

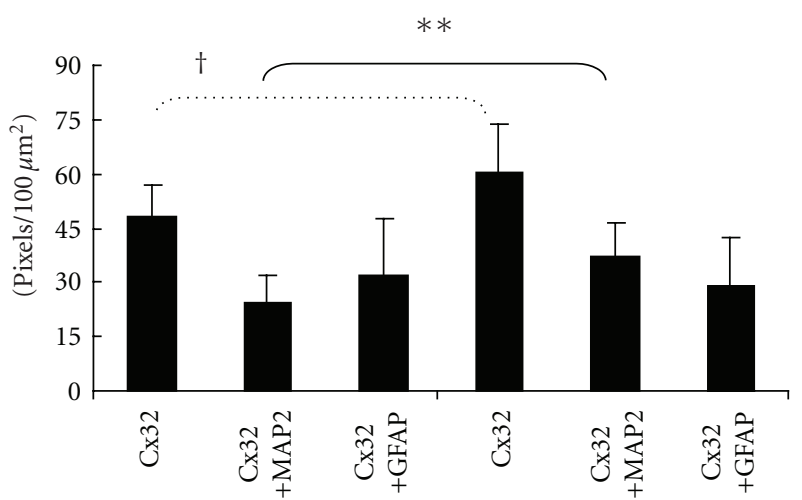

Intact area

Ischemic lesion

(g)

Figure 2: Cx32 immunofluorescent stainings indicate amplified expression in the ischemic lesion (b) compared to the intact area (a). Inset pictures of (c) and (d) represent double immunofluorescent staining with Cx32 (green) and MAP2 (red). Representative pictures in second row show the extracted area as yellow region of the double immunofluorescent staining from intact (c) and ischemic area (d). The yellow region which stands for Cx32 immunopositive area colocalized with MAP2 shows increased expression in the ischemic periphery. Inset pictures of (e) and (f) represent double immunofluorescent staining with Cx32 (green) and GFAP (red). Representative pictures in third row show the extracted area as yellow region of the double immunofluorescent staining from intact (e) and ischemic area (f). The yellow region which stands for Cx32 immunopositive area colocalized with GFAP seems similar level of the expression between normal and ischemic area. Black bars in graph (g) indicate the average counts of protein expressions. The $\mathrm{Cx} 32$ expression was relatively increased in the ischemic area compared to the intact area $(\dagger: P=.0510)$. The coexpression of $\mathrm{Cx} 32$ and MAP2 was significantly increased in the ischemic lesion as compared to the intact area $(* *: P<.01)$. However, there was no statistical difference in Cx32 and GFAP coexpression between intact and ischemic areas. Scale bar indicates $50 \mu \mathrm{m}$. 


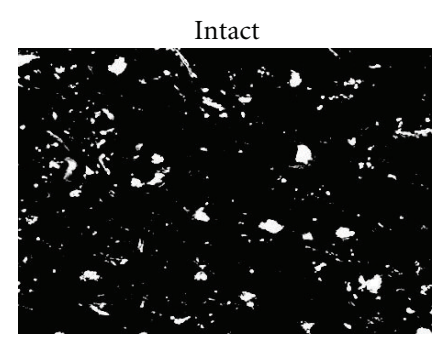

(a)

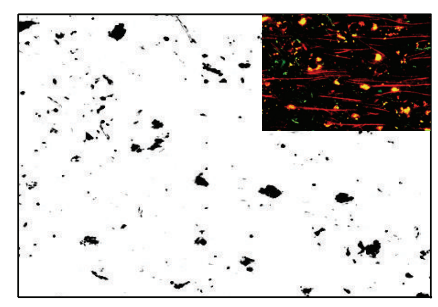

(c)

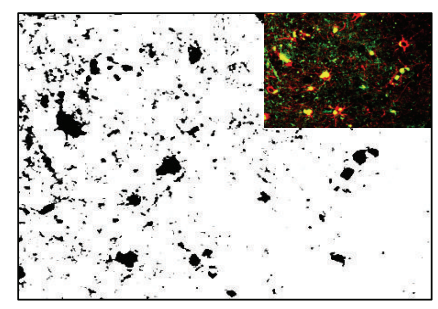

(e)

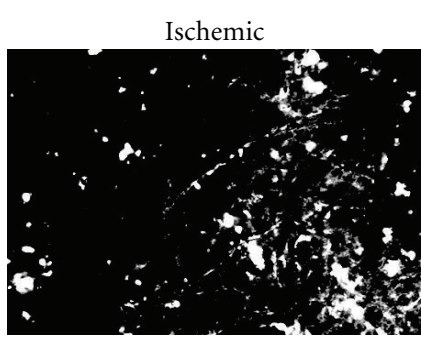

(b)

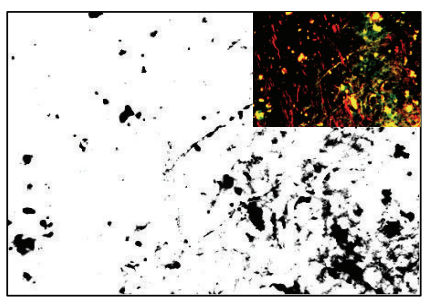

(d)

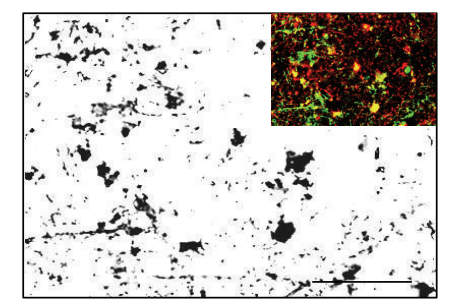

(f)

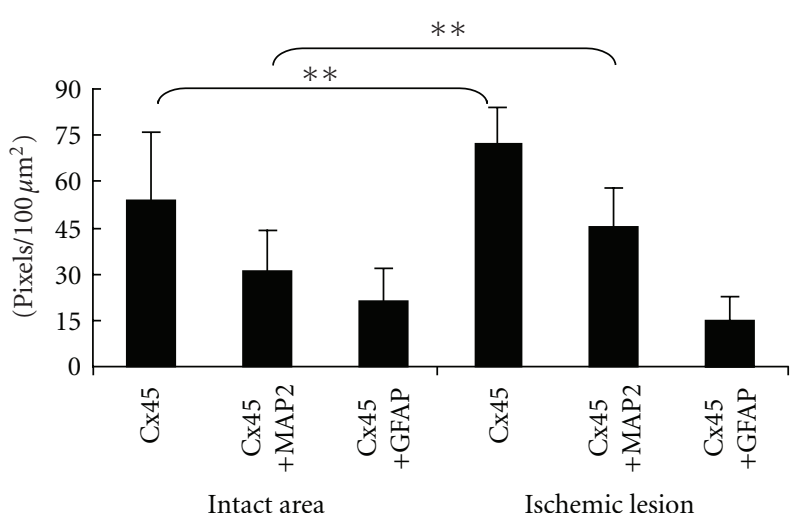

(g)

Figure 3: Cx45 immunofluorescent stainings indicate amplified expression in the ischemic lesion (b) compared to the intact area (a). Inset pictures of (c) and (d) represent double immunofluorescent staining with Cx45 (green) and MAP2 (red). Representative pictures in second row show the extracted area as yellow region of the double immunofluorescent staining from intact (c) and ischemic area (d). The yellow region which stands for Cx45 immunopositive area colocalized with MAP2 shows increased expression in the ischemic periphery. Inset pictures of (e) and (f) represent double immunofluorescent staining with Cx45 (green) and GFAP (red). Representative pictures in third row show the extracted area as yellow region of the double immunofluorescent staining from intact (e) and ischemic area (f). The yellow region which stands for Cx45 immunopositive area colocalized with GFAP shows no difference of expression between intact and ischemic area. Black bars in graph (g) indicate the average counts of protein expressions. The Cx45 expression was significantly increased in the ischemic area compared to the intact area. The coexpression of Cx45 and MAP2 was significantly increased in the ischemic lesion as compared to the intact area. However, no statistical alteration was observed in the amount of coexpression of Cx45 and GFAP between intact and ischemic regions. Scale bar indicates $50 \mu \mathrm{m} .{ }^{* *}: P<.01$. 


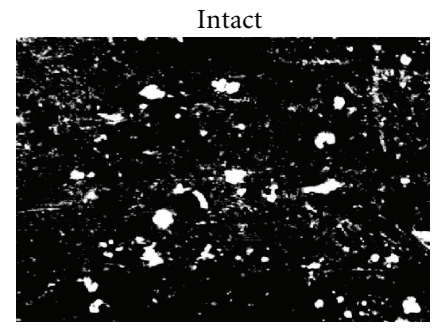

(a)

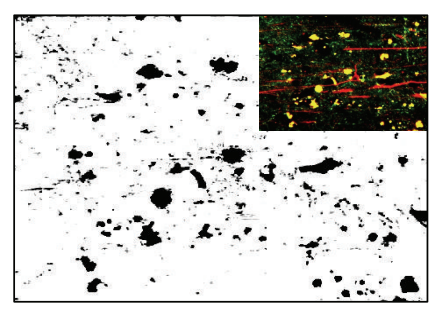

(c)

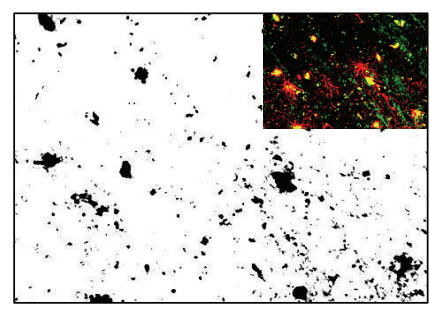

(e)

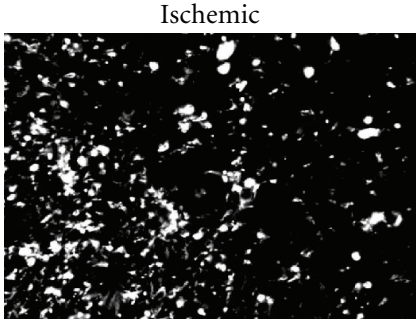

(b)

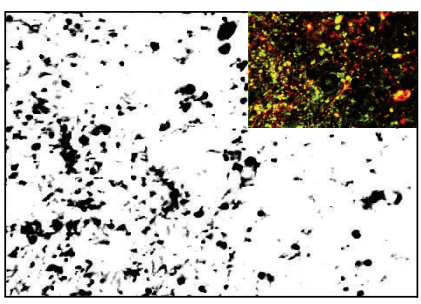

(d)

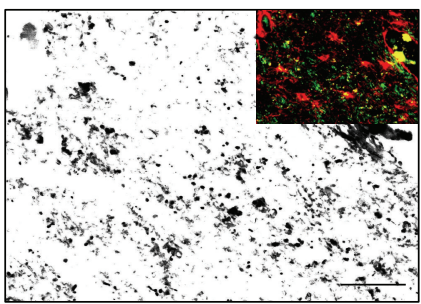

(f)

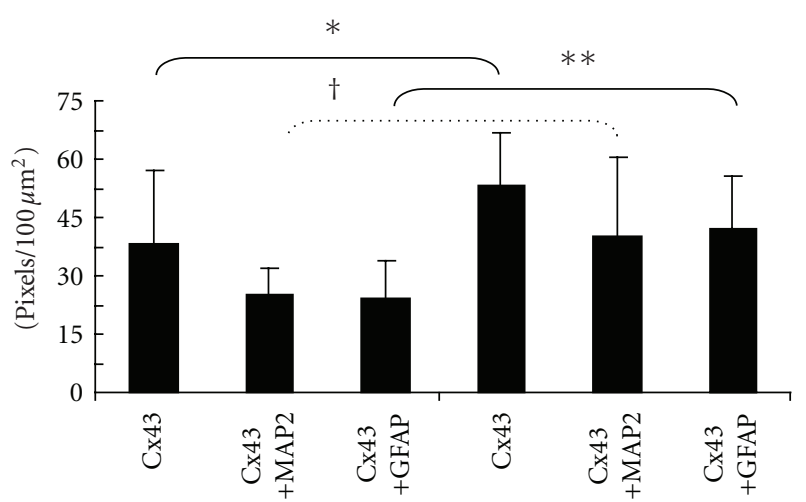

Intact area

Ischemic lesion

(g)

FIGURE 4: Cx43 immunofluorescent stainings indicate amplified expression in the ischemic lesion (b) compared to the intact area (a). Inset pictures of (c) and (d) represent double immunofluorescent staining with Cx43 (green) and MAP2 (red). Representative pictures in second row show the extracted area as yellow region of the double immunofluorescent staining from intact (c) and ischemic area (d). The yellow region which stands for $\mathrm{Cx} 43$ immunopositive area colocalized with MAP2 shows amplified expression in the ischemic periphery. Inset pictures of (e) and (f) represent double immunofluorescent staining with Cx43 (green) and GFAP (red). Representative pictures in third row show the extracted area as yellow region of the double immunofluorescent staining from intact (e) and ischemic area (f). The yellow region which stands for $\mathrm{Cx} 43$ immunopositive area colocalized with GFAP also shows increased expression level in ischemic lesion as compared to intact area. Black bars in graph (g) indicate the average counts of protein expressions. The Cx43 expression alone and the amount of coexpression with $\mathrm{Cx} 43$ or GFAP were significantly increased in the ischemic lesion compared to the intact area. The coexpression of Cx43 and MAP2 was increased in ischemic lesion compared to intact area $(\dagger: P=.0599)$. Scale bar indicates $50 \mu \mathrm{m} .{ }^{* *}: P<.01,{ }^{*}$ : $P<.05$. 


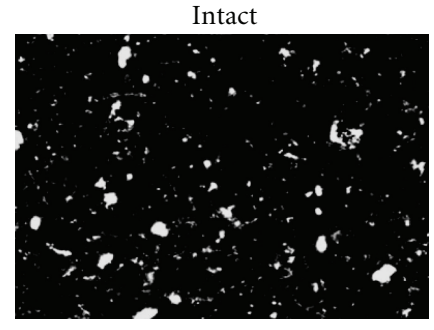

(a)

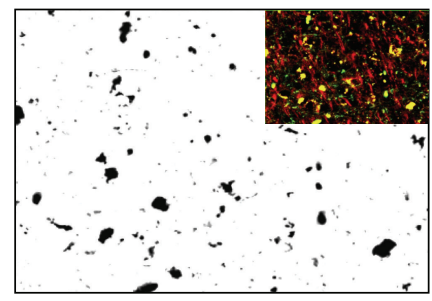

(c)

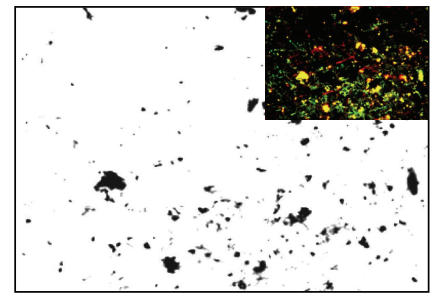

(e)

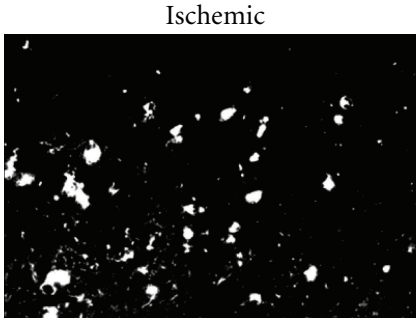

(b)

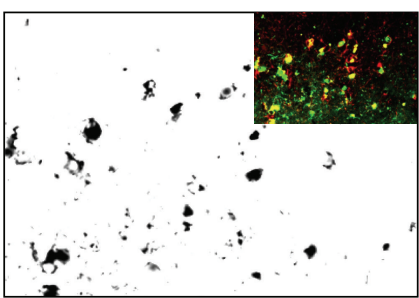

(d)

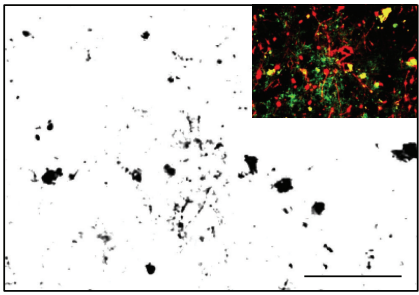

(f)

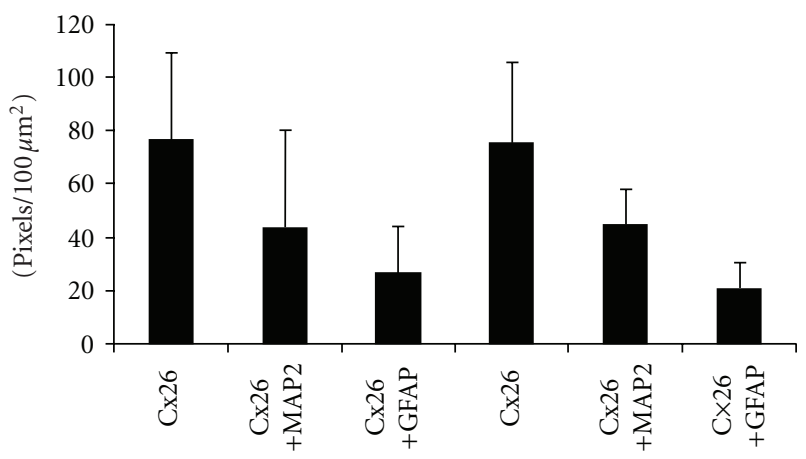

Intact area

Ischemic lesion

(g)

FIGURE 5: Cx26 immunofluorescent stainings show similar finding between intact area (a) and ischemic lesion (b). Inset pictures represent double immunofluorescent staining with Cx26 (green) and MAP2 (red) in (c) and (d) and with Cx26 (green) and GFAP (red) in (e) and (f). Representative pictures in second row show the extracted area as yellow region of the double immunofluorescent staining from intact (c) and ischemic area (d). The yellow region which stands for Cx26 immunopositive area colocalized with MAP2 shows the same amount of expression. The yellow region which stands for Cx26 immunopositive area colocalized with GFAP shows no difference of expression between intact and ischemic area. Black bars in graph (g) indicate the average counts of protein expressions. The Cx26 expression was not changed in the ischemic lesion as compared to the intact area. No statistical alteration was observed in the amount of coexpression of Cx26 and MAP2 nor Cx26 and GFAP between intact and ischemic regions. Scale bar indicates $50 \mu \mathrm{m}$.

astrocytes. In the future, gap junctions between different cell types should be directly observed through an electron microscope. Gap junctions of oligodendrocytes or microglia need further investigation in order to confirm the role of gap junctional intercellular communication following ischemic insult.
In conclusion, this is the first report that has revealed amplified expressions of $\mathrm{Cx} 32$ and $\mathrm{Cx} 45$ in MAP2 positive areas within the ischemic lesion from the view point of human brain pathology. Furthermore, Cx32 and Cx43 may have a neuroprotective role under ischemic conditions in the human brain. 


\section{References}

[1] A. Araque, V. Parpura, R. P. Sanzgiri, and P. G. Haydon, "Tripartite synapses: glia, the unacknowledged partner," Trends in Neurosciences, vol. 22, no. 5, pp. 208-215, 1999.

[2] R. Dermietzel and D. C. Spray, "From neuro-glue ('Nervenkitt') to glia: a prologue," Glia, vol. 24, no. 1, pp. 1-7, 1998.

[3] C. Giaume, C. Fromaget, A. El Aoumari, J. Cordier, J. Glowinski, and D. Gros, "Gap junctions in cultured astrocytes: singlechannel currents and characterization of channel-forming protein," Neuron, vol. 6, no. 1, pp. 133-143, 1991.

[4] C. C. G. Naus, J. F. Bechberger, S. Caveney, and J. X. Wilson, "Expression of gap junction genes in astrocytes and C6 glioma cells," Neuroscience Letters, vol. 126, no. 1, pp. 33-36, 1991.

[5] R. Siushansian, J. F. Bechberger, D. F. Cechetto, V. C. Hachinski, and C. C. G. Naus, "Connexin43 null mutation increases infarct size after stroke," Journal of Comparative Neurology, vol. 440, no. 4, pp. 387-394, 2001.

[6] T. Nakase, S. Fushiki, G. Sohl, M. Theis, K. Willecke, and C. C. G. Naus, "Neuroprotective role of astrocytic gap junctions in ischemic stroke," Cell Communication and Adhesion, vol. 10, no. 4-6, pp. 413-417, 2003.

[7] T. Nakase, G. Sohl, M. Theis, K. Willecke, and C. C. G. Naus, "Increased apoptosis and inflammation after focal brain ischemia in mice lacking connexin43 in astrocytes," American Journal of Pathology, vol. 164, no. 6, pp. 2067-2075, 2004.

[8] T. Nakase, Y. Yoshida, and K. Nagata, "Enhanced connexin 43 immunoreactivity in penumbral areas in the human brain following ischemia," Glia, vol. 54, no. 5, pp. 369-375, 2006.

[9] T. Nakase and C. C. G. Naus, "Gap junctions and neurological disorders of the central nervous system," Biochimica et Biophysica Acta, vol. 1662, no. 1-2, pp. 149-158, 2004.

[10] M. F. Anderson, F. Blomstrand, C. Blomstrand, P. S. Eriksson, and M. Nilsson, "Astrocytes and stroke: networking for survival?" Neurochemical Research, vol. 28, no. 2, pp. 293-305, 2003.

[11] G. S. Goldberg, V. Valiunas, and P. R. Brink, "Selective permeability of gap junction channels," Biochimica et Biophysica Acta, vol. 1662, no. 1-2, pp. 96-101, 2004.

[12] J. I. Nagy, W. Li, E. L. Hertzberg, and C. A. Marotta, "Elevated connexin43 immunoreactivity at sites of amyloid plaques in Alzheimer's disease," Brain Research, vol. 717, no. 1-2, pp. 173178, 1996.

[13] J. C. Vis, L. F. B. Nicholson, R. L. M. Faull, W. H. Evans, N. J. Severs, and C. R. Green, "Connexin expression in Huntington's diseased human brain," Cell Biology International, vol. 22, no. 11-12, pp. 837-847, 1998.

[14] J. E. Rash, T. Yasumura, K. G. V. Davidson, C. S. Furman, F. E. Dudek, and J. I. Nagy, "Identification of cells expressing Cx43, $\mathrm{Cx} 30, \mathrm{Cx} 26, \mathrm{Cx} 32$ and $\mathrm{Cx} 36$ in gap junctions of rat brain and spinal cord," Cell Communication and Adhesion, vol. 8, no. 46, pp. 315-320, 2001.

[15] J. I. Nagy, X. Li, J. Rempel, et al., "Connexin26 in adult rodent central nervous system: demonstration at astrocytic gap junctions and colocalization with connexin30 and connexin43," Journal of Comparative Neurology, vol. 441, no. 4, pp. 302-323, 2001.

[16] M. A. Filippov, S. G. Hormuzdi, E. C. Fuchs, and H. Monyer, "A reporter allele for investigating connexin 26 gene expression in the mouse brain," European Journal of Neuroscience, vol. 18, no. 12, pp. 3183-3192, 2003.
[17] A. Wallraff, R. Kohling, U. Heinemann, M. Theis, K. Willecke, and C. Steinhauser, "The impact of astrocytic gap junctional coupling on potassium buffering in the hippocampus," Journal of Neuroscience, vol. 26, no. 20, pp. 5438-5447, 2006.

[18] C. G. Bevans, M. Kordel, S. K. Rhee, and A. L. Harris, "Isoform composition of connexin channels determines selectivity among second messengers and uncharged molecules," The Journal of Biological Chemistry, vol. 273, no. 5, pp. 2808-2816, 1998.

[19] D. C. Spray and R. Dermietzel, "X-linked dominant CharcotMarie-Tooth disease and other potential gap-junction diseases of the nervous system," Trends in Neurosciences, vol. 18, no. 6, pp. 256-262, 1995.

[20] S. Fushiki and C. Kinoshita, "Intercellular communication through gap junctions," in Progress in Cell Research, Y. Kanno, K. Kataoka, Y. Shiba, Y. Shimazu, and T. Shimazu, Eds., vol. 4, pp. 239-243, Elsevier, Amsterdam, The Netherlands, 1995.

[21] R. Dermietzel, O. Traub, T. K. Hwang, et al., "Differential expression of three gap junction proteins in developing and mature brain tissues," Proceedings of the National Academy of Sciences of the United States of America, vol. 86, no. 24, pp. 10148-10152, 1989.

[22] G. S. Goldberg, A. P. Moreno, and P. D. Lampe, "Gap junctions between cells expressing connexin 43 or 32 show inverse permselectivity to adenosine and ATP," The Journal of Biological Chemistry, vol. 277, no. 39, pp. 36725-36730, 2002.

[23] K. Oguro, T. Jover, H. Tanaka, et al., "Global ischemia-induced increases in the gap junctional proteins connexin 32 (Cx32) and Cx36 in hippocampus and enhanced vulnerability of Cx32 knock-out mice," Journal of Neuroscience, vol. 21, no. 19, pp. 7534-7542, 2001.

[24] R. Dermietzel, M. Farooq, J. A. Kessler, H. Althaus, E. L. Hertzberg, and D. C. Spray, "Oligodendrocytes express gap junction proteins connexin32 and connexin45," Glia, vol. 20, no. 2, pp. 101-114, 1997.

[25] S. Maxeiner, O. Kruger, K. Schilling, O. Traub, S. Urschel, and K. Willecke, "Spatiotemporal transcription of connexin 45 during brain development results in neuronal expression in adult mice," Neuroscience, vol. 119, no. 3, pp. 689-700, 2003.

[26] G. Sohl, S. Maxeiner, and K. Willecke, "Expression and functions of neuronal gap junctions," Nature Reviews Neuroscience, vol. 6, no. 3, pp. 191-200, 2005.

[27] J. P. Neunuebel and M. J. Zoran, "Electrical synapse formation disrupts calcium-dependent exocytosis, but not vesicle mobilization," Synapse, vol. 56, no. 3, pp. 154-165, 2005.

[28] M. L. Cotrina, J. H.-C. Lin, A. Alves-Rodrigues, et al., "Connexins regulate calcium signaling by controlling ATP release," Proceedings of the National Academy of Sciences of the United States of America, vol. 95, no. 26, pp. 15735-15740, 1998.

[29] J. H.-C. Lin, H. Weigel, M. L. Cotrina, et al., "Gap-junctionmediated propagation and amplification of cell injury," Nature Neuroscience, vol. 1, no. 6, pp. 494-500, 1998.

[30] T. Nakase, S. Fushiki, and C. C. G. Naus, "Astrocytic gap junctions composed of connexin 43 reduce apoptotic neuronal damage in cerebral ischemia," Stroke, vol. 34, no. 8, pp. 19871993, 2003.

[31] C. Haupt, O. W. Witte, and C. Frahm, "Up-regulation of Connexin43 in the glial scar following photothrombotic ischemic injury," Molecular and Cellular Neuroscience, vol. 35, no. 1, pp. 89-99, 2007.

[32] C. Haupt, O. W. Witte, and C. Frahm, “Temporal profile of connexin 43 expression after photothrombotic lesion in rat brain," Neuroscience, vol. 144, no. 2, pp. 562-570, 2007. 


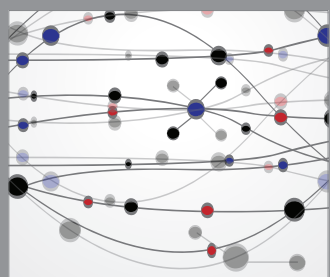

The Scientific World Journal
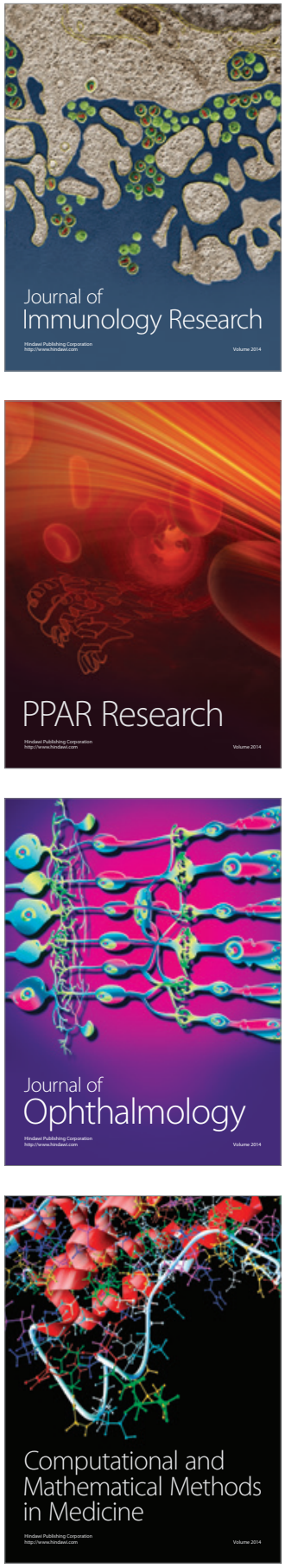

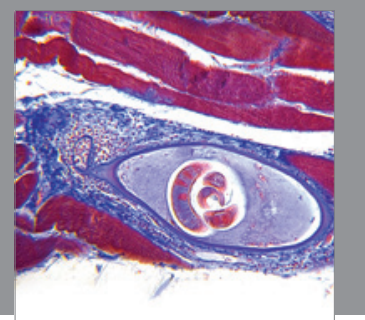

Gastroenterology

Research and Practice
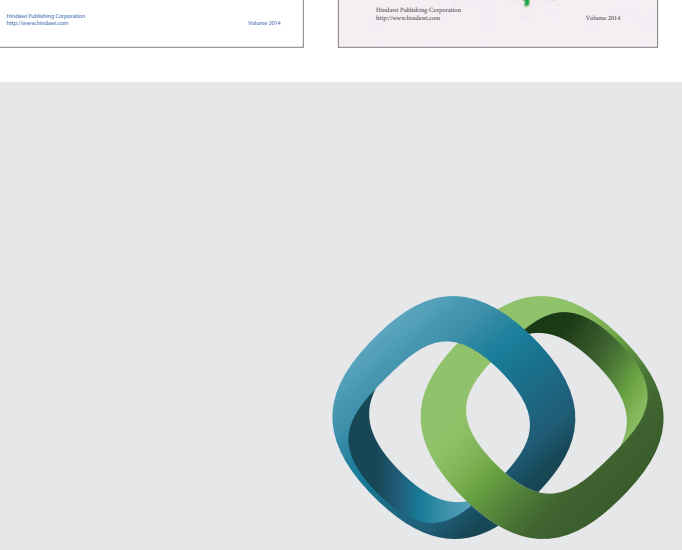

\section{Hindawi}

Submit your manuscripts at

http://www.hindawi.com
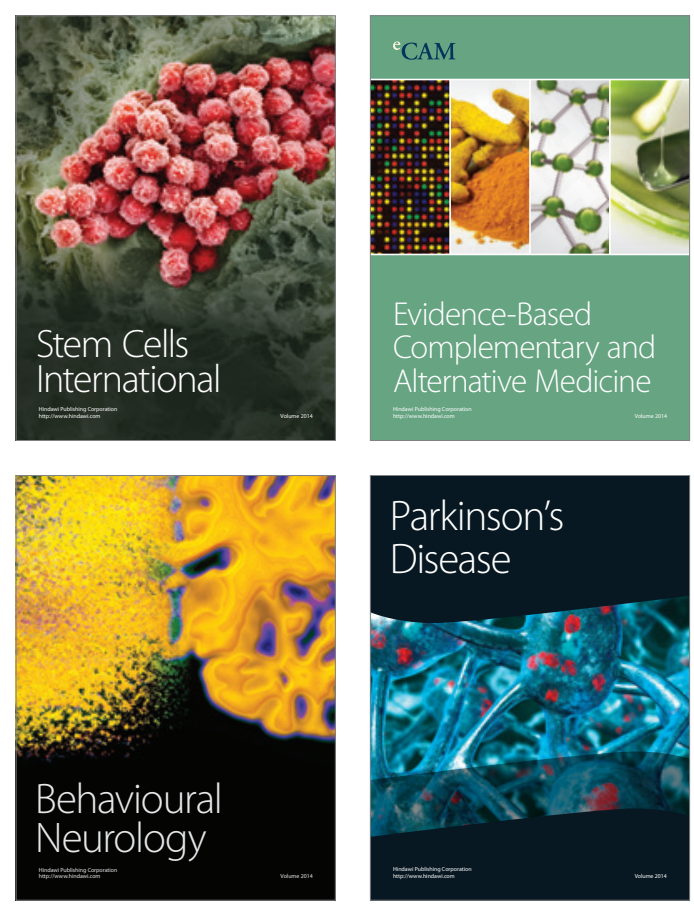

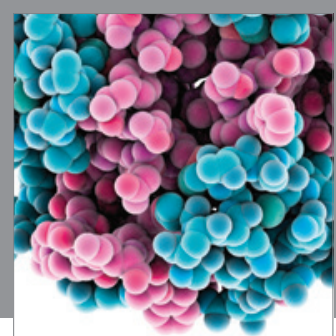

Journal of
Diabetes Research

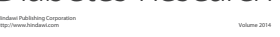

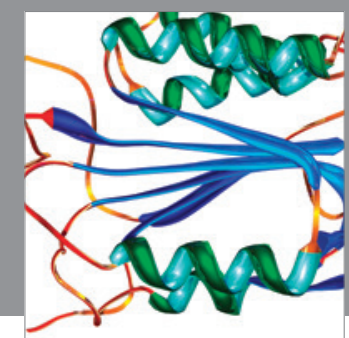

Disease Markers
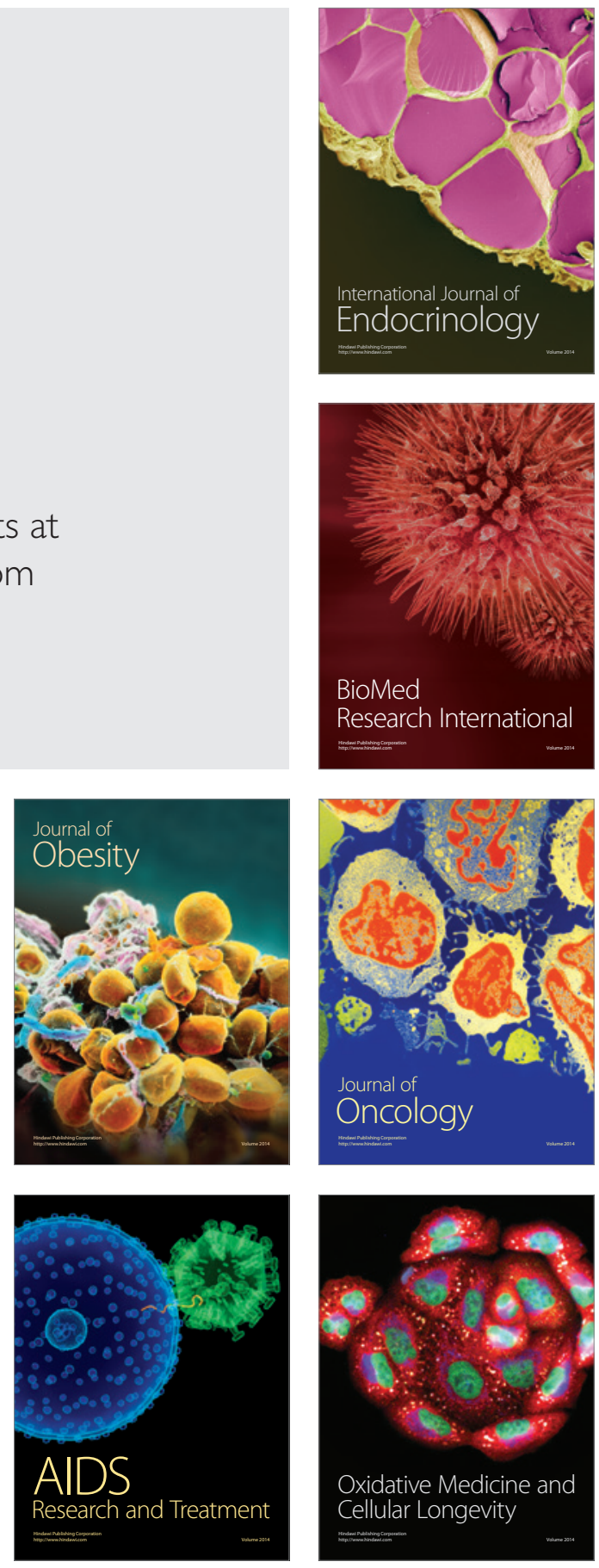